This is the accepted version of an article that was published in the International Journal of Primatology (36)

Doi: $10.1007 /$ s10764-015-9831-7

\title{
Lessons From the Past: Metabolic bone disease in historical captive primates
}

Milly Farrell,2*, Carolyn Rando 3 , Ben Garrod ${ }^{4,5}$

${ }^{1}$ Oxford Brookes University, Faculty of Health and Life Sciences, Oxford OX3 OBP, millyfarrell@brookes.ac.uk, 01865482890

${ }^{2}$ Royal College of Surgeons of England, 35-43 Lincoln's Inn Fields, London WC2A 3PE,

${ }^{3}$ Institute of Archaeology, University College London, WC1E 6BT,

${ }^{4}$ Department of Genetics, Evolution and Environment, University College London, London WC1E 6BT, UK, ${ }^{5}$ Institute of Zoology, Zoological Society of London, London, UK.

* Corresponding author

\section{ABSTRACT}

Primate welfare in captivity has significantly improved over the last century, due to the advances made in providing an adequate diet and environment. The skeletal collections of museums provide evidence of this shift in captive care, because metabolic disease caused by dietary deficiency or inappropriate surroundings can cause deformation to the hard tissues. The Royal College of Surgeons of England (RCS) holds a collection of 1,507 non-human primate skulls in its Odontological Collection, the majority donated prior to the mid twentieth century from various sources. We observed a recurring gross pathology in 51 of these skulls, noted in museum records as captive animals. In all cases, general bone thickening with decreased bone density is the main feature and primarily involves the bones of the maxillofacial region and mandible. We computer tomography scanned a sub-sample of these skulls to further investigate these pathological features. We compared the RCS historical collections and a more recent captive primate collection at the National Museum of Scotland. The findings suggest that a metabolic bone disease is the causative agent, with osteomalacia the likely diagnosis. Osteomalacia typically occurs due to malnutrition and/or insufficient ultraviolet light exposure and in this case reflects the inadequacy of zoo primate management during the late nineteenth and early twentieth centuries. Developments have since been made in captive animal welfare, due to improvements in nutrition and environment. Metabolic bone disease in primate captivity can be regarded as a lesson from the past.

\section{Key words}

Museum collections; Captivity; Osteomalacia ; Zoos 


\section{INTRODUCTION}

Animal welfare in zoos is a topic of marked interest in the modern literature, especially for threatened species (O'Regan and Kitchener 2005; Plesker and Zwerger 2002). Modern zoological parks take great care to maintain stimulating and healthy enclosures, as captive animals have less control than their wild counterparts with regards to food choice and behaviours, due to their somewhat restricted surroundings (Rees 2011; Wallace 2000). However, at the turn of the $20^{\text {th }}$ century, zoos were essentially arenas for human enjoyment, with little thought or care given to the conditions of the animals housed therein. This attitude, coupled with a profound lack of knowledge of nutrition, likely led to early deaths of many of the animals and a higher prevalence of certain diseases than would be normally seen in the wild. The reports of the time reflect this lack of understanding in nutrition and care, where the diseases present include a range of metabolic bone diseases (Plimmer 1910; Sutton 1884).

Metabolic bone disease is typically associated with a general loss of bone density (as is seen in osteopenia) or through the localised or systematic softening of the bones, as seen in both rickets and osteomalacia (Theiler 1934; Barker and Herbert 1972; Hosey et al. 2009). Metabolic bone disease results from a broad range of causative factors, although usually but not exclusively derived from an abnormality of minerals such as calcium or phosphorus, or through a lack of vitamin $D$ (Bernard et al. 1997). Metabolic bone diseases are typically associated with the following factors: incorrect diet, insufficient exposure to sunlight or (appropriate) artificial light and end-stage renal disease (Chesney 1984). Within zoological collections, several metabolic bone diseases are relatively common, with some occurring across taxa and others being restricted to a limited number of genera (Ruch 1959; Barker and Herbert 1972). These include:

1. Rickets, a developmental disease, referring to the softening of bone, with associated skeletal deformity and growth disturbance due to a deficiency in or a failure to metabolise vitamin D (Vieth 1999; Gartner and Greer 2003);

2. Osteomalacia, which refers to the softening of bone, specifically to the mineral loss of previously-mineralised tissue (i.e. in adults only), leaving a surfeit of organic matrix but not the gross pathological distortions of bone typically seen in rickets (Favus 2008) and is ultimately caused by a vitamin D deficiency (Bernard et al. 1997);

3. Fibrous osteodystrophy, which occurs when connective tissue replaces 'normally' mineralised tissue; a relationship also exists between fibrous osteodystrophy and nutritional secondary hyperparathyroidism (Jones et al. 1997);

4. Osteopenia, which describes moderate loss of bone density and mineral content (GonzalezReimers 2007; Seidlova-Wuttke 2008);

5. Osteoporosis, which results in severe loss of bone density and mineral content (Poole 2006; Santora, 2011).

Brooks and Blair (1904) first made the connection between metabolic bone disease and captivity in non-human primates in a report on two primate necropsies performed on behalf of the New York Zoological Society. Their report notes that no recorded instances of a similar disease in a wild environment existed, and goes as far as to suggest that there were "fewer cases occurring in the larger, more airy, and best lighted cages" (Brooks and Blair 1904; 117). Following this report, the 
twentieth century saw a steady improvement in primate husbandry knowledge and technique. Metabolic bone disease in captive primates has now become almost obsolete within both laboratory and zoological environments and where now recorded, occurrences are limited to those owned by private keepers (Hatt and Sainsbury 1998). The prevalence of metabolic bone disease amongst modern privately owned primates is likely a result "of ignorance on the part of the owner, as their diets are often inadequately balanced, and they receive insufficient exposure to ultraviolet light" (Hatt and Sainsbury 1998; 79).

In this study, we analyse skulls from documented collections for evidence of metabolic bone disease and carefully examine zoological reports dating to the same period, to better understand the conditions of primates and captive primate management during the turn of the twentieth century. We compare the occurrence of metabolic bone disease between Old and New World genera in the collections to determine whether one group is more prone to the disease than the other. We predict that those specimens donated around the turn of the twentieth century will show a higher propensity of metabolic bone disease than those derived from modern captive collections, as zoohusbandry knowledge and care has advanced significantly over the course of the last century (Hosey et al. 2009).

\section{MATERIALS AND METHODS}

Materials

The Royal College of Surgeons of England (RCS) currently houses four separate comparative anatomy collections, amassed over the past three centuries. A sizable proportion of the RCS primate material is held within the Odontological Collection, established over 150 years ago by dental surgeons, hence the high prevalence of cranial and absence of post cranial material within the collection. This collection contains over 11,000 skulls, skull portions, individual teeth and casts from an extensive range of species. There are 2321 specimens from 219 non-human primate species ( 23 genera), all of which are catalogued and available to access online (http://surgicat.rcseng.ac.uk/). Whilst the majority of the skulls now accessioned within the collection are wild caught animals, a small percentage originated from captive animals. Donations from zoos or individual collectors have added to this proportion of captive primates, with most of their original catalogue information retained alongside.

To obtain a modern day comparison to the RCS collections, we assessed the collections of the National Museum of Scotland (NMS). The NMS primate collection consists of animals sourced from recent zoological collections donated from the 1980s onwards.

To gain insight into zoological conditions in the early twentieth century, we accessed the pathology reports from the Zoological Society of London for evidence of disease, death rate, environment and diet. Since the late 19th century, yearly reports were compiled covering animal deaths at the Zoological Society of London (ZSL) and, where possible, relevant pathological findings. Complied by the resident ZSL veterinary pathologist, these reports covered all taxa within the zoo, with individual cases of interest receiving particular attention. The earliest available pathology reports from ZSL were basic lists of mortality (Plimmer 1909), loosely broken down at the taxa level. 


\section{Macroscopic analysis}

We used the complete catalogues for the RCS collections to identify the ratio of captive to wild-caught specimens. We then assessed these recently updated catalogues for occurrences of notable pathological bone conditions amongst both captive and non-captive specimens. MF individually examined all of these pathological skulls macroscopically and noted any pathological traits. MF wrote a detailed account for each instance of gross pathology, noting any variations in localisation of the pathology or differences in appearance.

MF and BG assessed the complete primate cranial collection of the NMS using a similar method of detailed macroscopic examination, noting any occurrence of pathology. Although no detailed catalogue exists for this material, the NMS provided us with a brief inventory of the species represented prior to our research visit.

\section{Statistical Analysis}

We used chi-squared tests (contingency matrix) and simple prevalence rates to analyse the data obtained from the two collections. First, we compared the two collections, considering presence/absence of disease only, to determine whether any differences between these two captive populations exist. Second, we analysed the RCS collection independently to test for differences between Old World and New World primates.

\section{Computed Tomography assessment}

We selected six specimens for CT scanning analysis, from three species in which the observed pathology was present. We used one complete adult male skull from each species Mandrillus sphinx, Macaca mulatta and Chlorocebus sabaeus to represent the typical pathological traits observed amongst the captive primates in the collection. To act as controls, we included one complete, non-pathological, adult male specimen from each species, chosen from the comparative anatomy material of the Odontological Collection.

We scanned each specimen on an i-CAT cone beam computed tomography (CBCT) unit at Cavendish Imaging, London; all scans took place on the same day and on the same machine. The unit was calibrated to the $\mathrm{Hi}-\mathrm{Fi}$ setting $(90 \mathrm{Kv}, 214.20 \mathrm{mAs})$ to obtain the maximum radiation dosage. This was necessary to provide the clearest images and to best differentiate between different densities. Axial slices were taken at $0.25 \mathrm{~mm}$ increments, with an imaging window of $640 \times 640 \mathrm{~mm}$; the total field of view was $16 \mathrm{~cm}$. We extracted DICOM images from the scanning unit and imported these into a dedicated CT/CBCT viewing software, SimPlant Pro 12.03 (designed for planning dental implant surgery) for virtual reconstruction. The software reformats the original axial slices into coronal and sagittal slices, and allows for the creation and use of virtual 3D models.

Once imported into SimPlant, the programme converts each of the volume elements (voxels) into a specific grey value unit (or Hounsfield Unit). These units represent the different densities present in the scanned object. In a conventional clinical CT scanner, there is a set and well- 
defined scaling system for what each Hounsfield unit represents. For example, air has a value of 1000 (varies slightly depending on the reformatting program), while water is valued at 0 (Lagravere et al., 2008; Mah et al., 2010); bone densities typically fall within the range of 250-500, or greater. However, no such standardised scaling system yet exists for CBCT scanners and, as such, the grey values represented are difficult to interpret and it is almost impossible to compare results with other scanners, conventional or CBCT (Mah et al., 2010). For the purposes of this study, the crucial component was being able to compare between the control and pathological specimens scanned, and establish the different relative densities, as well as the internal structural changes associated with the observed pathological condition.

\section{RESULTS}

\section{ZSL Reports}

In 1909, 'congestion of the lungs' attributed to 84 primate deaths, with approximately $50 \%$ of these animals suffering from 'severe, terminal rickets' at the time of death. Over the next decade, numerous primate deaths were attributed to either rickets or a whole suite of rare, tropical and in some cases taxonomically impossible conditions including goundou, type I neurofibromatosis and leontiasis ossia.

Between the end of the 19th century and the late 1920s, the ZSL pathological reports were sporadic and non-standardised in nature. Apart from the suggestion of several varied, exotic and in some instances implausible diagnoses, numerous primate deaths presented with 'rickets-like' symptoms (Hamerton 1929; 1931). On occasion, this loosely termed 'rickets' appears as an actual cause of death, rather than as a secondary pathology, as in earlier reports. It is apparent from the earlier reports that there was little or no distinction made between any of the metabolic bone diseases and 'rickets' was often used as an overarching pathological term. In the 1931 zoo pathology report, 'rickets' was for the first time differentiated from osteomalacia, when the deaths of one Kuhl's owl monkey (Aotus felinus) and one galago (Otolemur garnettii) were attributed to "systemic osteomalacia and associated broncho-pneumonia" (Hamerton 1931; 543). Although this was the first mention of osteomalacia being both present and acting as a causative factor in ZSL primate deaths, it appears that either the diagnosis or the use of the term is used loosely for the proceeding few years and was used almost interchangeably with rickets, in addition to the more 'exotic' and largely inaccurate diagnoses. In a further suspected case of gondou (or leontiasis ossia) in a hamadryas baboon (Papio hamadryas), its described symptoms suggests a severe case of osteomalacia, with the animal displaying "symmetrical overgrowth of cancellous bone in the maxillae", growth from the right superior maxilla extending into and distorting the nasal cavity and with "bones of the calvarium being thicker than normal" (Hamerton 1934; 399).

\section{Macroscopic Analysis}

Amongst the 1507 complete captive primate skulls in the RCS collection, 53 display symptoms of a gross pathology of the skull, largely localised in the maxillae and (on occasion) affecting the mandible, nasals and frontal (Table 1). All of these affected animals are from donations 
prior to 1950 , and the majority are from zoo post-mortems during the 1920 s. Captive-held animals presenting the above-described pathological signs come from a wide range of primate species.

In specimens displaying recurring pathological traits, we observed thickening of bone at varying degrees in the cranium, including the frontal (particularly concentrated around glabella), parietal and temporal bones with some specimens displaying thickening in the zygomatic bones extending anteriorly into the maxilla (Fig 1 ). Thickening of the cranial bones seems to occur primarily in the frontal region, extending posteriorly. Figure 2 indicates extensive thickening of the cranial vault, a feature only visible in this one specimen macroscopically given its previous sectioning shortly after acquisition. Generally, there is little evidence of pathology in the occipital bones although four specimens show slight swelling of the bone, which may be related to the onset of the condition. In cases where we noted a combined swelling of bone in both the maxilla and mandible, there is evidence of this effecting the eruption and positioning of the teeth.

In the cranio-facial region, we observed thickening of both the maxilla and mandible, although this was present at varying stages and not necessarily in conjunction with thickening of the cranial vault. In those specimens indicating the pathology, the maxillae assumed an appearance of general swelling, and occasionally this localised bone swell occurs when there is no thickening of any other bones of the skull and may therefore reflect the early onset of the pathology.

In the specimens showing gross pathology, the orbital margins seem very rounded with a softened orbital edge and where present, the nasal conchae and palate also display signs of thickening. There is evident widening of the sutures and, on occasion, the facial sutures (frontalzygomatic and temporal process) are particularly widened and almost disarticulated. This can also been seen along the sagittal suture in specimens where extensive distortion of the bones is evident.

We observed an increase in bone porosity in all specimens where bone thickening has occurred, with the size and frequency increasing to large pitted gullies across the bones surface, particularly evident along the sagittal suture, glabella and maxillae. The mandible also shows an increase in porosity, with thickened bone appearing swollen and spongy with loss of surface definition. This aspect of the pathology has made the bone extremely fragile and friable, with some exposure of the inner trabecular bone in several affected specimens. Although store environment may be a factor here, the loss of cortical bone and evident surface porosity in many of the observed captive specimens are attributed to pathology alone.

The mandible is also a commonly affected element, either exclusively or in conjunction with general thickening across the skull. In a number of specimens, the mandibular condyles seem underdeveloped, as though the articular surface has failed to ossify with exposure of the trabecular bone (Fig. 1). A large number of pathological specimens have post-mortem damage to the mandibular condyles and as a result, on occasion the condyles are missing. We could not therefore determine whether underdevelopment of the articular surfaces of the mandibular condyles is common in all affected specimens. We observed no pathological examples of probable metabolic bone disease in any specimens originating from 'known wild' animals held within the RCS collection (table 1), or in any of the specimens from the modern NMS primate collection. 
We found a significant difference in the frequency of metabolic bone disease in captive skulls between the RCS collection (53 of 671 skulls, 7.9\%) and the NMS ( 0 of 158; 1 way Chi-square test, $\left.\chi^{2}=13.33, p=0.003,1 \mathrm{df}\right)$. Among the pathological specimens in the RCS collection, we found no significant difference between Old World primates (i.e. Cercopithecus, Chlorocebus, Erythrocebus, Macaca, Mandrillus, Papio and Eulemur) and New World primates (i.e. Alouatta, Ateles, Callithrix, Cebus and Aotus) when comparing these two separate groups (Chi-square test, $\chi^{2}=0.017, p=0.8959$, $1 \mathrm{df})$.

\section{CT results}

The results of the CT scan analysis supported those reported for the macroscopic observations. All pathological skulls presented with marked thickening of the bones of the cranial vault and an expansion of the maxillae and surrounding bone. In all three species selected for CT assessment, we noted a similar pattern between the control and pathological specimens. In the control specimens, there was a clear delineation between the outer and inner tables of the cranial bones (considering specifically the occipital, parietals, temporals and frontal), with the cortical bone displaying higher density values relative to the internal medullary space (Fig. 3). All the pathological specimens showed a notable increase in the thickness of the cranial bones, but had a decrease in relative bone density and virtually no delineation between the inner/outer tables; rather, the bone was uniform in density and there was no evidence of an internal space (Fig. 3-4). There was also a reduction in overall bone density in the pathological specimens, as noted in the bones of the cranial vault and facial region, which were markedly less dense in the pathological group. In addition to the changes within the cranium, we noted marked porosity (macroscopically) in the mandibular condyle; we also observed this in the CT scans, with the porosity penetrating the external cortical layer.

\section{DISCUSSION}

We observed a recurring gross pathology in 51 skulls in the RCS collection, noted in museum records as captive animals. Through macroscopic observation and CT assessment, we determined that the main pathological traits observed include thickening of the bones across the cranium, widening of the sutures and increased bone porosity of both cranium and mandible. The CT scan results revealed the extent of the thickening in the affected specimens which are all known to have been captive prior to the mid-twentieth century. Given that there are no clinical signs of a transmissible nature to the pathology, such as the florid markings of a bacterial infection, and based on an extensive review of the literature, we surmise that this reoccurring pathology falls under the category of metabolic bone disease, as an umbrella term used to cover a wide variety of diseases linked to the disruption in the animal's metabolism (Theiler 1934; Chesney 1984; Ullrey 2003). Specimens displaying these pathological changes were all captive pre-1950 (in the Odontological Collection of the RCS), which suggests a lack of understanding of animal management at the time, also evident from the yearly reports from the ZSL.

The ZSL reports suggest a range of diagnoses for the pathology, including goundou, type I neurofibromatosis and leontiasis ossia. Goundou is a very rare, tropical disease resulting from yaws 
and a spirochete infection geographically restricted (at the time) to humans in western Africa, which was cited on several occasions within the reports (Hamerton 1929; Hamerton 1931). Rarely observed in non-human primates, there is some evidence of goundou occurring in great apes (Cousins 2008). Type I neurofibromatosis (formerly von Recklinghausen's disease), is an autosomal dominant disorder characterized by various neurological symptoms, severe cutaneous tumours and skeletal symptoms including scoliosis and sphenoid wing dysplasia (Williams et al. 2009). This disease is only found in humans and therefore an incorrect diagnosis. Leontiasis ossia, characterised by an overgrowth of the facial and cranial bones was also posited as a diagnosis on several occasions in the reports. Leontiasis ossia is typically secondary to conditions such as Paget's disease and hyperparathyroidism, although the term is now used for cases of chronic inflammatory disease of the maxilla (Barker and Herbert 1972).

Although often causing serious and gross clinical conditions, metabolic bone diseases are commonly treatable and reversible. They are rare in wild populations, and commonly associated with a species expanding its home range into an area of 'suboptimal habitat', characterised with a reduced availability of calcium-rich food (Fidgett and Dierenfeld 2007). The results of our macroscopic assessment of the RCS collection support this. The RCS contained both wild and captive animal specimens, but we found no indication of the pathology in any of the wild specimens (Table 1). Furthermore, we found no significant differences between Old and New World primates, suggesting that the occurrence of the disease is likely to be related to the captive origins of the affected animals. In captive environments, metabolic bone disease is reported to be a common factor and according to historical records was a near ubiquitous disorder in zoo environments pre1940 (Sutton 1884; Barker and Herbert 1972).

A complex relationship exists between vitamin $D$, ultraviolet $B$ (UVB) radiation and minerals in the body (Hosey et al. 2009). Whilst wild primates meet their vitamin D requirements through their diet or through the exposure to UV radiation, captive animals typically need husbandry intervention to enable them to synthesise and process it effectively. Zoological parks typically attribute nutritional problems in captive primates to the ingestion of non-food items, the provisioning of incorrect foods, obesity or malnutrition (Hosey et al. 2009). The nutritional content of wild fruits differs from those provided for captive primates; those used in zoological parks are typically low in calcium (Joslin 2003) and differences such as these historically have led to a high prevalence of metabolic bone diseases in some captive primate species. Our results support this historical shift in the occurrence of metabolic bone disease, as we found no evidence of metabolic bone disease in a modern captive collection.

Primates are still able to develop specific metabolic bone diseases, such as osteomalacia, even if they are provided with an adequate level of vitamin $D$ in their diet, which is reportedly due to insufficient access to UVB light (Favus 2008). Vitamin D (specifically vitamin $D_{3}$ ) forms in the skin when there are appropriate levels of UV exposure (Bernard et al. 1997). The conversion of 7dehydrocholesterol (the vitamin $D$ precursor in the skin) to previtamin $D_{3}$ generally occurs between 285-315nm (UVB middle bandwidth); previtamin $\mathrm{D}_{3}$ then thermally converts to vitamin $\mathrm{D}_{3}$ (Bernard, 1997). Whilst most zoological parks now use windows and skylights made from specially-constructed glass-like material for the effective transmission of UVA and UVB radiation levels (Bernard 1997), in the past the glass used often did not permit adequate levels of UV radiation to enter an animal's enclosure. Coupled with little or no access to outdoor enclosures (and full UVB radiation), this glass 
was also very likely to be significant in the historically high prevalence of metabolic bone diseases in captive primates in zoos. In the mid-late 19th century, the Zoological Society of London developed primate enclosures (the well-known 'Monkey Houses') in the style of a conservatory (Sclater 1870), so that they were able to provide as much light as possible and to emulate the circumstances under which the animals lived in the wild (Sclater 1870; see Fig. 5). Whilst appearing bright and providing adequate access to natural light, not fitting the enclosures with the appropriate UV-permeating glass (highly likely given the period) would have affected the health of the enclosed animals and may contribute to the pathological evidence obtained from the zoological reports.

The results of our analysis of the two collections (historical and modern) support the evidence obtained from the zoological reports and the shift in captive primate management. In 1929 -1930, a ZSL report made a key observation, noting "animals which are accommodated in the open air throughout the year suffer less acute inflammation of the lungs than those kept indoors" (Hamerton 1931; 532). This indicates that not only were some primates kept indoors permanently, but that zoo staff were becoming aware that housing and husbandry directly impacted animal health, heralding a turning point in zoo welfare management (at least at ZSL). The report proceeds to identify some varying success with artificial UV lamps for the treatment of observed metabolic bone disease, where the withdrawal of its use led to death in several instances. One further advance was the observation that not only were certain taxa (canids and primates) more prone to metabolic bone diseases, but also that animals were beginning to be given vitamin supplements for dietary disorders and that "time [spent] outside was beneficial for avoiding rickets" (Hamerton 1931; 533). This shift in the husbandry methods applied to captive primate care resulted in the decrease of the occurrence of metabolic bone disease, which no longer became a pathological norm. In the 1930s, both dietary and hygienic management at ZSL and many other zoos improved drastically. A significant result was fewer zoo deaths across the whole zoo per annum. This resulted in the subsequent adaptation of primate enclosures enabling the inhabitants to spend time both indoors and outdoors, and also the provision of adequate indoor UVB lighting (Hamerton 1936; Guillery 1993). In modern zoological parks, a combination of effective dietary care (including dietary supplements) and either direct solar exposure, artificial UVB light sources or UVB-conductive glass has seen the incidence of osteomalacia (and several other metabolic bone diseases) in primates greatly reduced (Favus 2008). Gillespie et al. (2001) found that through the use of corrective measures in housing, such as UV lamps, direct exposure to sunlight or the use of UV-permeable skylights, vitamin $\mathrm{D}_{3}$ for animals housed indoors can be brought to levels similar to those in outdoor enclosures (or even in wild animals).

\section{CONCLUSION}

We infer key developments in captive primate management over the course of the last century, by assessing the occurrence of metabolic bone disease in two primate skeletal collections; one comprising specimens donated in the late nineteenth and early twentieth centuries (from the Royal College of Surgeons of England) and the other containing modern specimens (from the National Museum of Scotland). We found the disease in a wide range of primate taxa, suggesting that it was not due to failures in taxon-specific husbandry, but that fundamental welfare practices were not in place in early primate enclosures. We observed no signs of osteomalacia, or any other metabolic bone diseases, within the modern captive collection (assembled from 1980s to the present), demonstrating the modernization of captive primate care. A combination of meeting the 
dietary needs and providing adequate UVB requirements means that metabolic bone diseases such as osteomalacia are no longer a problem amongst primates in zoological collections and can be regarded as a vital lesson from the past.

\section{ACKNOWLEDGEMENTS}

We would like to thank the following people and organisations for their contribution to this study; Dr Andrew Kitchener (National Museum of Scotland), Dr Sam Alberti and John Carr (Royal College of Surgeons of England), Cavendish Imaging and the Faculty of Dental Surgery for their support. We would also like to extend our sincere gratitude to the editor and our reviewers for their invaluable comments and suggestions.

\section{REFERENCES}

Barker, M.J.M. and Herbert, R.T. (1972). Diseases of the Skeleton. In R.N.T-W-Fienes (Ed.), Pathology of Simian Primates (pp. 433-519). Basel: S.Karger pub.

Bernard JB. (1997). Vitamin D and ultraviolet radiation: meeting lighting needs for captive animals. In Nutrition Advisory Group Handbook Fact Sheet 002. Accessed 17 June 2013.

[http://nagonline.net/wp-content/uploads/2014/01/NAG-FS002-97-Vit-D-JONI-FEB-24-2002MODIFiED.pdf]

Brooks, H. and Blair, W.R. (1904). Eighth Annual Report of the New York Zoological Society for 1903. New York: Crow Press.

Chesney, R.W. (1984). Metabolic Bone Diseases. Pediatrics in Review, 5(8), 227-237.

Clauss M., Dierenfeld, E.S. (2007). The Nutrition of Browsers. In Fowler, M.E. and Miller, R.E. (Eds.) Zoo and Wild Animal Medicine: current therapy (6th edition). St. Louis, MO: Saunders (Elsevier).

Favus, M.J. (2008). Primer on the Metabolic Bone Diseases and Disorders of Mineral Metabolism (4th edition). Philadelphia: American Society for Bone and Mineral Research.

Fidgett, A.L., Dierenfeld, E.S. (2007). Minerals and Stork Nutrition. In Fowler, M.E. and Miller, R.E. (Eds.) Zoo and Wild Animal Medicine: current therapy (6th edition). St. Louis, MO: Saunders (Elsevier).

Gartner, L.M. and Greer, F.R. (2003). Prevention of Rickets and Vitamin D deficiency: new guidelines for vitamin D intake. Pediatrics, 111, 908-10.

Gillespie, D., Frye, F.L., Stockham, S.L. and Fredeking, T. (2001). Blood Values in Wild and Captive Komodo Dragons (Varanus komodoensis). Zoo Biology, 19, 495-509.

Guillery, P. (1993). The Buildings of London Zoo. London: Royal Commission on the Historical Monuments of England. 
Gonzalez-Reimers, E. (2007). Quantitative computerized tomography for the diagnosis of osteopenia in prehistoric skeletal remains. Journal of Archaeological Science, 34(4), 554-561.

Hatt, J.M. and Sainsbury, A.W. (1998). Unusual case of metabolic bone disease in a common marmoset (Callithrix jacchus). Veterinary Record, 143, 78-80.

Hamerton, A.E. (1929). Report on the Deaths Occurring in the Society's Gardens During the Year 1928. Proceedings Zoological Society of London - Yearbook, 99(1), 49-59.

Hamerton, A.E. (1931). Report on the Deaths Occurring in the Society's Gardens During the Year 1930. Proceedings Zoological Society of London - Yearbook, 101(2), 527-555.

Hamerton, A.E. (1934). Report on Deaths Occurring in the Society's Gardens During the Year 1933. Proceedings Zoological Society of London - Yearbook, 104(2), 389-422.

Hamerton, A.E. (1936). Report on the Deaths Occurring in the Society's Gardens During the Year 1935. Proceedings Zoological Society of London - Yearbook, 106, 659-686.

Hamerton, A.E. (1937). Report on the Deaths Occurring in the Society's Gardens During the Year 1936. Proceedings Zoological Society of London - Yearbook, 107, 443-474.

Hamerton, A.E. (1941- 1942). Report on the Deaths Occurring in the Society's Gardens During the Years 1939-1940. Proceedings Zoological Society of London - Yearbook, 111 (1-2), 151-184.

Hosey, G., Melf, V. and Pankhurst, S. (2009). Zoo Animals: Behaviour, Management and Welfare. Oxford: Oxford University Press.

Jones, T.C., Hunt, R.D. and King, N.W. (1997). Veterinary Pathology (6th edition). Baltimore: Williams and Wilkins.

Joslin, J.O. (2003). Other Primates Excluding Great Apes. In Fowler, M.E. and Miller, R.E. (Eds.) Zoo and Wild Animal Medicine (5th edition). Philadelphia: Saunders.

Lagravere, M.O., Carey, J., Ben-Zvi, M., Packota, G.V. and Major, P.W. (2008). Effect of object location on the density measurement and Hounsfield conversion in a NewTom $3 \mathrm{G}$ cone beam computed tomography unit. Dentomaxillofacial Radiology, 37, 305-308.

Mah, P., Reeves, T.E. and McDavid, W.D. (2010). Deriving Hounsfield units using grey levels in cone beam computed tomography. Dentomaxillofacial Radiology, 39, 323-335.

Otto, A.W. 1831. A Compendium of Human and Comparative Pathological Anatomy. London: B. Fellowes.

O'Regan, H.J. and Kitchener, A.C. (2005). The effects of captivity on the morphology of captive, domesticated and feral mammals. Mammal Review, 35, 215-230.

Plesker, R. and Zwerger, C. (2002). Rickets, osteomalacia and osteoporosis in an indoor non-human primate facility. Primate Report, 62, 69-78.

Plimmer, H. G. (1910). Report on the Deaths which occurred in the Zoological Gardens during 1909. Proceedings of the Zoological Society of London, 80, 131-136. 
Poole, K.E.S. (2006). Osteoporosis and its management. British Medical Journal, 333(7581), 12511256.

Preacher, K.J. (2001). Calculation for the chi-square test: An interactive calculation tool for chisquare tests of goodness of fit and independence [Computer software]. Available from: http://quantpsy.org

Rees, P.A. (2011). An Introduction to Zoo Biology and Management. Chichester: Wiley-Blackwell, John Wiley \& Sons Ltd.

Rewell, R. E. (1948). Report of the Pathologist for the Year 1947. Proceedings of the Zoological Society of London, 118, 501-514.

Ruch, T.C. (1959). Diseases of Laboratory Primates. Philadelphia, London: Saunders.

Santora, L. and Skolbekken, J.A. (2011). From Brittle Bones to Standard Deviations: The Historical Development of Osteoporosis in the Late Twentieth Century. Science, Technology \& Human Values, 36(4), 497-521.

Schmidt, R.E., Reavill, D.R. and Phalen, D.N. (2008) Pathology of Pet and Aviary Birds. lowa: WileyBlackwell.

Sclater, P.L. (1870). Guide to the Gardens of the Zoological Society of London (24th edition). London: Bradbury, Evans and Co.

Seidlova-Wuttke, D. (2008). Orchidectomized (orx) marmoset (Callithrix jacchus) as a model to study the development of osteopenia/osteoporosis. American Journal of Primatology, 70(3), 294-300.

Sutton, J.B. (1884). Observations on Rickets in Captive and Wild Animals. Journal of Anatomy, 18, 363-387.

Theiler, A. (1934). Osteodystrophic Disease of Domesticated Animals. Veterinary Journal, 90, 159175.

Ullrey, D.E. (2003). Metabolic Bone Diseases. In Fowler, M.E. and Miller, R.E. (eds.) Zoo and wild animal medicine: current therapy (5th edition) (pp. 749-756). Philadelphia, PA: Elsevier (Saunders).

Vieth, R. (1999). Vitamin D supplementation, 25 hydroxyvitamin D concentrations, and safety. The American Journal of Clinical Nutrition, 69(5), 842-56.

Williams, V.C., Lucas, J., Babcock, M.A., Gutmann, D.H., Korf, B. and Maria, B.L. (2009).

Neurofibromatosis type 1 revisited. Pediatrics, 123, 124-133. 